\title{
Model Based Architecting and Construction of Embedded Systems (ACES-MB 2009)
}

\author{
Stefan Van Baelen ${ }^{1}$, Thomas Weigert ${ }^{2}$, Ileana Ober $^{3}$, \\ Huascar Espinoza ${ }^{4}$, and Iulian Ober ${ }^{3}$ \\ 1 K.U.Leuven - DistriNet, Belgium \\ Stefan.VanBaelen@cs.kuleuven. be \\ 2 Missouri University of Science and Technology, USA \\ weigert@mst.edu \\ 3 University of Toulouse - IRIT, France \\ \{Ileana.Ober, Iulian.Ober\}@irit.fr \\ 4 CEA - LIST, France \\ hdeo@hotmail.com
}

\begin{abstract}
The second ACES-MB workshop brought together researchers and practitioners interested in model-based software engineering for real-time embedded systems, with a particular focus on the use of models for architecture description and domain-specific design, and for capturing non-functional constraints. Eleven presenters proposed contributions on domain-specific languages for embedded systems, the Architecture Analysis and Design Language (AADL), analysis and formalization, semantics preservation issues, and variability and reconfiguration. In addition, a lively group discussion tackled the issue of combining models from different Domain Specific Modeling Languages (DSMLs). This report presents an overview of the presentations and fruitful discussions that took place during the ACES-MB 2009 workshop.
\end{abstract}

\section{Introduction}

The development of embedded systems with real-time and other critical constraints raises distinctive problems. In particular, development teams have to make very specific architectural choices and handle key non-functional constraints related to, for example, real-time deadlines and to platform parameters like energy consumption or memory footprint. The last few years have seen an increased interest in using model-based engineering (MBE) techniques to capture dedicated architectural and non-functional information in precise (and even formal) domain-specific models in a layered construction of systems.

The Second Workshop on Model Based Architecting and Construction of Embedded Systems (ACES-MB 2009) brought together researchers and practitioners interested in all aspects of model-based software engineering for real-time embedded systems. The participants discussed this subject at different levels, from model specification languages and analysis techniques to model-based implementation, deployment and reconfiguration. The workshop was attended by 47 registered participants coming from 19 different countries. 


\section{Workshop Contributions}

The keynote [2] was given by Prof. Marco Di Natale from the Scuola Superiore Sant'Anna in Pisa, Italy, who discussed semantics preservation issues in the design and optimization of software architectures for automotive systems.

Architecture selection and design optimization are critical stages of the Electronics/Controls/Software (ECS)-based vehicle design flow. In automotive systems design, complex functions are deployed onto the physical hardware and implemented in a software architecture consisting of a set of tasks and messages. Several optimizations of the software architecture design were presented, including the definition of the task periods, the task placement and the signal-tomessage mapping. In addition, the assignment of priorities to tasks and messages were automated in order to meet end-to-end deadlines and to minimize latencies.

Architecture selection can be accomplished by leveraging worst case response time analysis within an optimization framework. Suggestions on how to use stochastic or statistical analysis to improve the approach were provided. Semantics preservation issues impose additional constraints on the optimization problem, but also reveal very interesting tradeoffs between memory and time/ performance. The need to deal with heterogeneous models and standards, such as AUTOSAR (AUTomotive Open System ARchitecture) in the automotive industry, further complicates the scenario.

7 full papers and 3 short papers had been accepted for the workshop, see [1]. A synopsis of each presentation is given below. Extended versions of articles [1] and $[12$ are included in this workshop reader.

3 presents SOPHIA, a modelling language that formalizes safety-related concepts (e.g., accident models), and explores strategies to implement SOPHIA as a complementary modelling language to SysML.

[4 describes a prototype modeling language for the design of networked control systems using passivity to decouple the control design from network uncertainties. The resulting designs are by construction more robust with respect to platform effects and implementation uncertainties.

[5] presents a formal specification of the design models used in COMDES-II, a component-based framework for distributed control systems, featuring an open architecture and predictable operation under hard real-time constraints.

6. proposes a systematic method for Worst-Case Execution-time (WCET) analysis that make Simulink/Stateflow model information (e.g., infeasible executions) available to static timing analysis tools.

7] presents a methodology and tool for building and translating AADL systems into a distributed application. This allows runtime analysis to fully assess system viability, and to refine and correct the behavior of the system using BIP.

8] investigates the iterative model-driven design process for complex embedded systems, which suffers from a delay between applying changes to the model and obtaining information about the resulting properties of the system, and identifies opportunities for a novel, integrating research domain on AADL.

[9] presents a methodology for the specification and analysis of critical embedded systems, based on an architectural design language that enables modeling 
of both software and hardware components, timed and hybrid behavior, faulty behavior and degraded modes of operation, error propagation, and recovery.

10] focuses on finding performance problems when use cases are added to (a family of) products, and proposes an automated algorithm that generates performance models by combining use case models, and an approach for performance optimization by adding flow control elements into the system design.

11] integrate variability approaches from Software Product Lines (SPL) into Domain-Specific Languages (DSLs) like Matlab / Simulink, in order to specify variability, configure products and to resolve element dependencies. High-Order Transformations derive the variability mechanisms (as a generated model transformation) from the meta-model of the DSL.

[12] supports the combination of robustness and flexibility in automotive systems through the integration of reconfiguration capabilities into AUTOSAR, supporting the management of complex system at the architectural level.

\section{Summary of the Workshop Discussions}

The workshop was divided into 4 sessions: domain-specific languages for embedded systems, the Architecture Analysis and Design Language (AADL), analysis and formalization, and variability and reconfiguration. After each session, a group discussion was held on topics and issues raised during the session presentations. The following integrates and summarizes the conclusions of the discussions.

\section{AUTOSAR}

The automotive industry is challenged to distribute an increasing number of functions, which are enabled through an increasing number of smart sensors and actuators, onto a descreasing number of Electronic Control Units (ECUs). The AUTOSAR architecture is a key element for the automotive industry to decouple the software functions from the ECUs. The main challenges for the automotive embedded system industry are (1) how to perform the mapping of functions onto ECUs, and (2) how to preserve the semantics of these functions. The focus here is on the timing analysis to obtain an adequate mapping of the tasks to the resources. Currently no structured hierarchical methodology is used, but the allocation is performed using a bottom-up approach through a number of iterations. Regarding communication between ECUs, the CAN (Controller Area Network) bus is rather easy to deal with, while the FlexRay protocol is more complex. In most cases no formalized architectural frameworks are used on top of AUTOSAR. The trickiest part for the timing analysis is to get the correct Worst Case Execution Time (WCET) bound for safe and precise resource optimization, hereby avoiding overestimations due to infeasible paths. Further research on obtaining accurate WCET information is therefore necessary.

\section{Combining models from different DSMLs}

There is a need for using different DSMLs during the development of embedded systems. The main challenges hereby are (1) to combine such models and 
map identical representations onto a single concept, (2) to obtain semantic compatibility, and (3) to guarantee and preserve consistency between these models. The UML-MARTE profile seems to be too complex for ordinary developers and end users for expressing non-functional system properties; they require a simple language that is easily understandable. In addition, there can be a need to use several DSMLs, and the results expressed in these domainspecific models must be compatible and consistent. Three approaches for connecting domain-specific models were discussed. First, explicit semantic interfaces can be defined to connect heterogeneous models. The link between AUTOSAR, Simulink, and Modelica is an example. Second, an underlying semantic framework can be used to map the domain-specific models onto a universal model. Such approach also has to deal with concepts present in the DSML that cannot easily be mapped onto the underlying framework. UMLMARTE could be a suitable candidate as an underlying supporting semantic framework for building domain-specific models that could be mapped and integrated on top of UML-MARTE. Finally, a third approach is to restrict a general model to a specific subset, for instance by defining a restrictive UML profile. SysML can be seen as an example of defining a restrictive DSML on top of UML. In practice, we will have to combine all three approaches during embedded systems development. There is a need to utilise heterogenerous models that either are subsets of UML-MARTE, will be mapped onto it, or will be interfaced with it.

The correctness by construction approach is very powerful, but very difficult to realise when using heterogeneous models. If one can define the right abstractions and refine them, then one no longer needs to reopen the component box when connecting to them. This works fine for some combinations (e.g., from a synchronous reactive system description to a time-triggered architecture), but not for all combinations (e.g., to an event-driven architecture). One solution is to use only time-triggered architectures, and take the potential inefficiency as a fact, since reliability and composability might be more important than efficiency. This may be a good approach at least in some domains, such as aerospace and automotive, but may not apply in other domains, such as consumer electronics. This approach is best suited for a one-pass development process, while in practice incremental development is often used. The developer models part of the functionality, defines an architecture based on estimates, analyses and simulates the application, and then tries to obtain more concrete and precise estimates during later stages of the development. Iteratively, the developer can go back to change the behavior and functional and/or physical architecture at higher levels based on this refined information. As such, one starts with a simplified version of the system that gradually is adjusted and reshaped until a suitable system description is obtained.

\section{Reconfiguration}

Reconfiguration is often a key element even in safety-critical embedded systems. It is used to obtain a graceful degradation in an FDIR (Fault Detection, 
Isolation and Recovery/Reconfiguration) approach, enabling continuous operation under degraded conditions. Often the failing system cannot be shut down but must reach a stable state as soon as possible. It is not clear if such safe state can always be reached, and if so in which timeframe. Therefore the aerospace industry heavily relies on redundancy in order to avoid system failures. Similarly, the introduction of steer-by-wire in the automotive industry is not a matter of computational power or price but rather of reliability and culture, since there is not yet the same level of culture about safety and reliability as in the aerospace industry.

\section{Acknowledgements}

We thank all workshop participants for the lively discussions. We especially thank our keynote speaker, Prof. Marco Di Natale, for his inspiring presentation. We also thank the workshop steering committee and program committee members for their support. This workshop was supported by the ARTIST2 Network of Excellence on Embedded Systems Design (http://www.artist-embedded.org) and by the EUREKA-ITEA project SPICES (http://www.spices-itea.org).

\section{References}

1. Van Baelen, S., Weigert, T., Ober, I., Espinoza, H. (eds.): Second International Workshop on Model Based Architecting and Construction of Embedded Systems. CEUR Workshop Proceedings, Aachen, Germany. CEUR, vol. 507 (2009)

2. Di Natale, M.: Semantics preservation issues in the design and optimization of SW architectures for automotive systems. In: [1], p. 9

3. Cancila, D., Terrier, F., Belmonte, F., Dubois, H., Espinoza, H., Gérard, S., Cuccuru, A.: SOPHIA: a modeling language for model-based safety engineering. In: [1], pp. 11-25

4. Eyisi, E., Porter, J., Hall, J., Kottenstette, N., Koutsoukos, X., Sztipanovits, J.: PaNeCS: A modeling language for passivity-based design of networked control systems. In: [1], pp. 27-41

5. Angelov, C., Sierszecki, K., Guo, Y.: Formal design models for distributed embedded control systems. In: [1], pp. 43-57

6. Tan, L., Wachter, B., Lucas, P., Wilhelm, R.: Improving timing analysis for Matlab Simulink/Stateflow. In: [1], pp. 59-63

7. Chkouri, M.Y., Bozga, M.: Prototyping of distributed embedded systems using AADL. In: [1], pp. 65-79

8. Langsweirdt, D., Vandewoude, Y., Berbers, Y.: Towards intelligent tool-support for AADL based modeling of embedded systems. In: [1], pp. 81-85

9. Bozzano, M., Cimatti, A., Katoen, J.P., Nguyen, V.Y., Noll, T., Roveri, M.: Modelbased codesign of critical embedded systems. In: [1], pp. 87-91

10. Ersfolk, J., Lilius, J., Muurinen, J., Salomäki, A., Fors, N., Nylund, J.: Design complexity management in embedded system design. In: [1], pp. 93-106

11. Botterweck, G., Polzer, A., Kowalewski, S.: Using higher-order transformations to derive variability mechanisms for embedded systems. In: [1], pp. 107-121

12. Becker, B., Giese, H., Neumann, S., Schenck, M., Treffer, A.: Model-based extension of AUTOSAR for architectural online reconfiguration. In: [1], pp. 123-137 